\title{
Expression of microRNA-30a-5p in drug-resistant and drug-sensitive ovarian cancer cell lines
}

\author{
JIN LIU ${ }^{1,2}$, XIAOHUA WU ${ }^{1,3}$, HONGMEI LIU $^{4}$, YIJUAN LIANG ${ }^{2}$, XINPING GAO $^{2}$, \\ ZHIHUI CAI ${ }^{2}$, WEIMING WANG ${ }^{2}$ and HUI ZHANG ${ }^{2}$ \\ ${ }^{1}$ Department of Gynecology, Hebei Medical University, Shijiazhuang, Hebei 050017; \\ ${ }^{2}$ Department of Gynecology, Affiliated Hospital of Hebei University, Baoding, Hebei 071000; \\ ${ }^{3}$ Department of Gynecology, Bethune International Peace Hospital, Shijiazhuang, Hebei 050000; \\ ${ }^{4}$ Department of Molecular Medicine, Hebei University, Baoding, Hebei 071000, P.R. China
}

Received April 15, 2015; Accepted June 7, 2016

DOI: $10.3892 / \mathrm{ol} .2016 .4831$

\begin{abstract}
The present study aimed to explore the expression of microRNA (miRNA or miR) in drug-resistant and drug-sensitive ovarian cancercell lines, and to seek the potential therapeutic target of ovarian cancer drug-resistant mechanism in order to improve drug resistance by altering miRNA levels. The drug-resistant characteristics of SKOV3/DDP, SKOV3, COC1/DDP and COC1 cell lines were studied. The miRNAs that were differentially expressed between cisplatin-resistant cells and its parental cells in ovarian cancer were screened with a miRNA chip. The effect of miRNAs was detected, and their drug-resistant mechanism was investigated by transfection and 3-(4,5-dimethylthiazol-2-yl)-2,5-diphenyltetrazolium bromide methods. Among the expression screening of miRNAs, 41 mRNAs, including Homo sapiens (hsa)-miR-30a-5p and hsa-miR-34c-5p, were highly expressed in the drug-resistant cells, whereas 44 miRNAs, including hsa-miR-96-5p and hsa-miR-200c-3p, were lowly expressed. The expression levels of hsa-miR-30a-5p in two types of ovarian cancer chemotherapy-resistant cell lines were significantly higher than those in chemotherapy-sensitive cell lines, which was associated with ovarian cancer chemotherapy resistance. In conclusion, high expression of miRNA-30a-5p was able to promote cell growth and colony forming ability, and enhance cell migration and invasion. Thus, miRNA-30a-5p is expected to become a meaningful novel target for ovarian cancer resistant treatment.
\end{abstract}

Correspondence to: Dr Xiaohua Wu, Department of Gynecology, Bethune International Peace Hospital, 398 Zhongshan West Road, Shijiazhuang, Hebei 050000, P.R. China

E-mail: xiaohuawucn@163.com

Key words: ovarian cancer, microRNA, microRNA-30a-5p, drug resistance

\section{Introduction}

Ovarian cancer is a common malignant tumor seriously threatening women's lives and health. Its mortality rate ranks first among all gynecological tumors (1). In the past 10 years, along with the wide development of cytoreductive surgery, novel chemotherapy drugs have emerged unceasingly (1). Since chemotherapy plans are constantly improving, the treatment of ovarian cancer has greatly improved in recent years, and the patients' quality of life has improved significantly as a result (2). However, the 5-year survival rate is still $30-50 \%$; thus, chemotherapy has not obviously improved the overall survival rate (3). One of the main reasons for this observation is primary or secondary drug resistance during chemotherapy. Previous studies revealed that tumor drug-resistant mechanisms included oncogene activation, anti-oncogene inactivation, reduced intracellular drug concentration, drug target molecular changes, metabolism detoxification, enhanced DNA damage repair function and inhibition of tumor cells apoptosis, which is a comprehensive result of polygenic, multiple factors and multiple steps (2). Ovarian cancer drug resistance has a great impact on the prognosis of ovarian cancer. Therefore, studying the occurrence and development mechanisms of ovarian cancer drug resistance in order to reverse this process could greatly improve the chemotherapy effect on ovarian cancer. To identity the regulatory mechanisms responsible for ovarian cancer chemotherapy drug resistance in order to improve the tumor sensitivity to chemotherapeutic drugs (particularly in recurrent tumors) is one of the effective ways to improve the survival rate of ovarian cancer patients. The current study identified multiple microRNAs (miRNAs or miRs) that were differentially expressed in tumor tissue and serum of ovarian cancer patients. These miRNAs have been reported to be important in the occurrence and progression of ovarian cancer, and are expected to be used in early diagnosis, prognosis, recurrence and treatment of ovarian cancer (4-6).

miRNAs are small non-coding RNAs with gene regulation function, which are 20-25 nucleotides-long and are processed from a hairpin-structure precursor (7). Previous studies demonstrated that miRNAs are mainly involved in the post-transcriptional control of eukaryotic gene expression by 
inhibiting the translation of their target gene or mediating the degradation of their target gene messenger RNA (mRNA) (7). miRNAs participate in cell proliferation, differentiation, apoptosis and cell cycle regulation processes. In recent years, a growing number of studies demonstrated that miRNAs are involved in multiple processes, including tumor occurrence, development, invasion, metastasis and prognosis (8). miRNAs are important in the occurrence and development of breast, colorectal, lung, ovarian and liver cancer, as well as other malignant tumors (9). Kinose et al (10) noticed that the expression levels of miR-15a and miR-16 were decreased in ovarian cancer tissues and cell lines. miR-15a and miR-16 directly acted on the proto-oncogene (B-cell specific moloney leukemia virus insertion site 1) Bim-1 and played a role as anti-oncogenes by inhibiting the expression of Bim-1 (10). Previous studies also demonstrated that miR-21 was abnormally highly expressed in ovarian cancer tissues, and was closely associated with the differentiation of tumor cells and lymph node metastasis, suggesting that miR-21 may promote the development of ovarian cancer as an oncogene (11). These studies indicated that the occurrence and development processes of ovarian cancer were closely associated with miRNA levels $(11,12)$.

miRNAs are closely associated with the drug sensitivity of numerous tumors. Among them, miRNA let-7i was the first member of the miRNA family identified to be associated with tumor drug sensitivity, and was observed to play an indirect anti-tumor role by increasing the cell sensitivity to chemotherapeutic drugs (13). Lee et al (14) demonstrated that the expression of let-7i was decreased in platinum-resistant ovarian tumor cells, and downregulation of the expression of let-7i could increase ovarian cancer resistance to cisplatin, suggesting that let-7i could be used as a chemical marker to evaluate the prognosis of ovarian cancer. However, the specific mechanism of chemotherapy drug resistance remains unclear. Nurkkala et al (15) demonstrated that inhibition of phosphatase and tensin homolog (PTEN)-targeted regulation by an AKT inhibitor or miR-214 directly could reduce the miR-214-mediated survival rate and cisplatin-resistance through PTEN/AKT signaling in PTEN/AKT-induced ovarian cancer drug-resistant cells. The above methods could increase chemotherapy drug sensitivity of ovarian cancer patients, which could be used in the treatment of recurrent and chemotherapy-resistant ovarian cancer in order to improve the treatment of ovarian cancer.

In the present study, using the drug-resistant characteristics of SKOV3/DDP, SKOV3, COC1/DDP and COC1 cells, the differences in miRNA expression profiles between the SKOV3/DDP, SKOV3, COC1/DDP and COC1 cell lines were compared. miRNA expression in drug-resistant and drug-sensitive ovarian cancer cell lines was detected. The potential therapeutic target of the ovarian cancer drug-resistant mechanism and the possible improvement of drug resistance was investigated at the miRNA level.

\section{Materials and methods}

Cells and chip. The drug-resistant and drug-sensitive ovarian cancer cell lines SKOV3 and COC1 cell lines (SKOV3/DDP, drug-resistant; SKOV3, drug-sensitive;

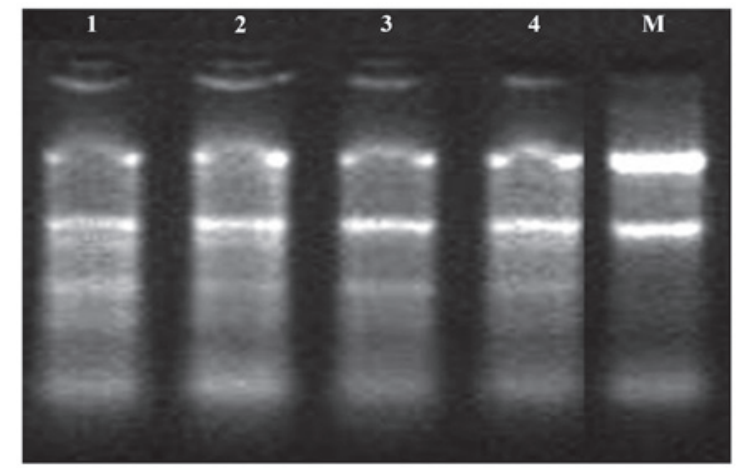

Figure 1. Determination of messenger RNA extraction quality. 1, SKOV3 cells; 2, SKOV3/DDP cells; 3, COC1 cells; 4, COC1/DDP cells; M, marker.

COC1/DDP, drug-resistant; COC1, drug-sensitive) were used in the present study. SKOV3/DDP and SKOV3 cells were purchased from the Cancer Institute and Hospital, Chinese Academy of Medical Sciences (Beijing, China). SKOV3 cells and SKOV3/DDP cells were cultivated in RPMI-1640 culture medium containing $10 \%$ fetal bovine serum $\left(\mathrm{Gibco}^{\circledR}\right.$; Thermo Fisher Scientific, Inc., Waltham, MA, USA) without penicillin or streptomycin under $5 \% \mathrm{CO}_{2}$ and $37^{\circ} \mathrm{C}$ saturated humidity. These cells exhibited adherent growth. COC1/DDP and $\mathrm{COC} 1$ cells were purchased from the Wuhan University cell library, and cultivated in RPMI-1640 culture medium containing $10 \%$ fetal bovine serum without penicillin or streptomycin under $5 \% \mathrm{CO}_{2}$ and $37^{\circ} \mathrm{C}$ saturated humidity. These cells displayed suspended growth. The drug-resistant SKOV3/DDP and COC1/DDP maintenance concentration of cisplatin (Qilu Pharmaceutical Co. Ltd., Jinan, China) was $1 \mu \mathrm{g} / \mathrm{ml}$.

A total of 924 probes (data from Sanger miRNA database miRBase 12.0, August 2012; www.pageinsider.com/microrna. sanger.ac.uk) were designed for a chip experiment aiming to analyze 677 human, 292 rat and 461 mice mature miRNAs, using the mammalian miRNA chip V3 (Beijing Bo'ao Hengxin Biotechnology Co., Ltd., Beijing, China). The probe was arrayed on $75 \times 25-\mathrm{mm}$, chemically modified glass slides with chip microarray SmartArrayer ${ }^{\mathrm{TM}}$ (CapitalBio Corporation, Beijing, China). Each probe was repeated three times.

Reverse transcription-quantitative polymerase chain reaction $(R T-q P C R)$. Total cell miRNA was extracted using the TaqMan probe method (Thermo Fisher Scientific, Inc.) and extracted using mirVana miRNA Isolation kit (Ambion; Thermo Fisher Scientific, Inc.). miRNA was detected with the Applied Biosystems 7300 Real-Time PCR System (Thermo Fisher Scientific, Inc.) with the following cycling conditions: $95^{\circ} \mathrm{C}$ for $10 \mathrm{~min}$, and 40 cycles of $95^{\circ} \mathrm{C}$ for $15 \mathrm{sec}$ and $60^{\circ} \mathrm{C}$ for $30 \mathrm{sec}$, followed by melt-curve analysis.

Cell transfection. Cell transfection was performed with Lipofectamine 2000, according to the manufacturer's protocol (Invitrogen; Thermo Fisher Scientific, Inc.). The final concentration of the miRNA-transfected molecules Homo sapiens (hsa)-miR-30a-5p precursor molecule pre-hsa-miR-30a-5p, hsa-miR-30a-5p inhibitory molecule anti-hsa-miR-30a-5p and the corresponding negative controls pre-scramble and 


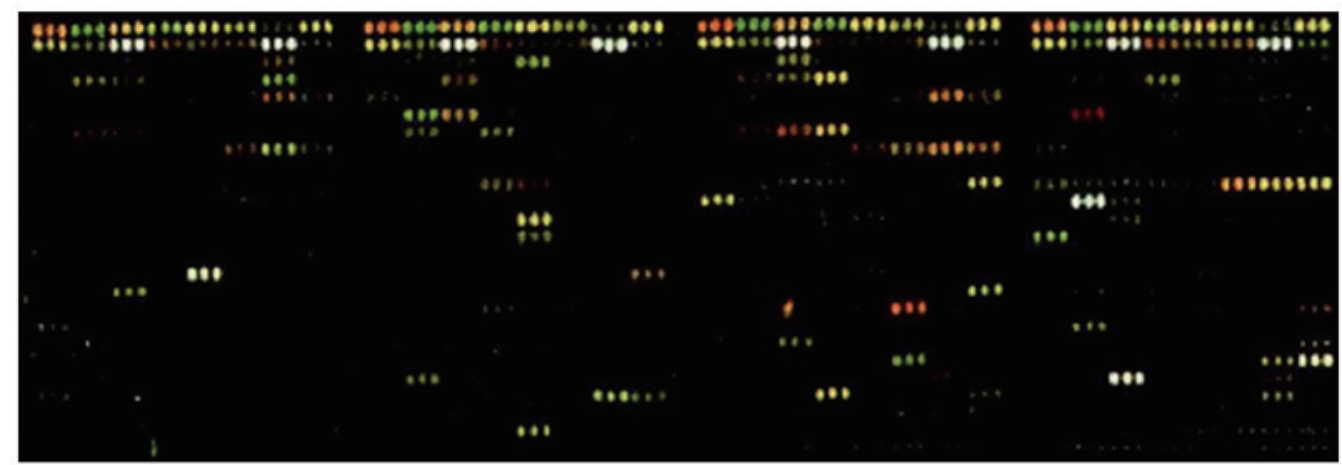

Figure 2. microRNA chip hybridization results. Red represents Cy5; green represents Cy3; yellow represents that the instensity of Cy3 and Cy5 was the same.

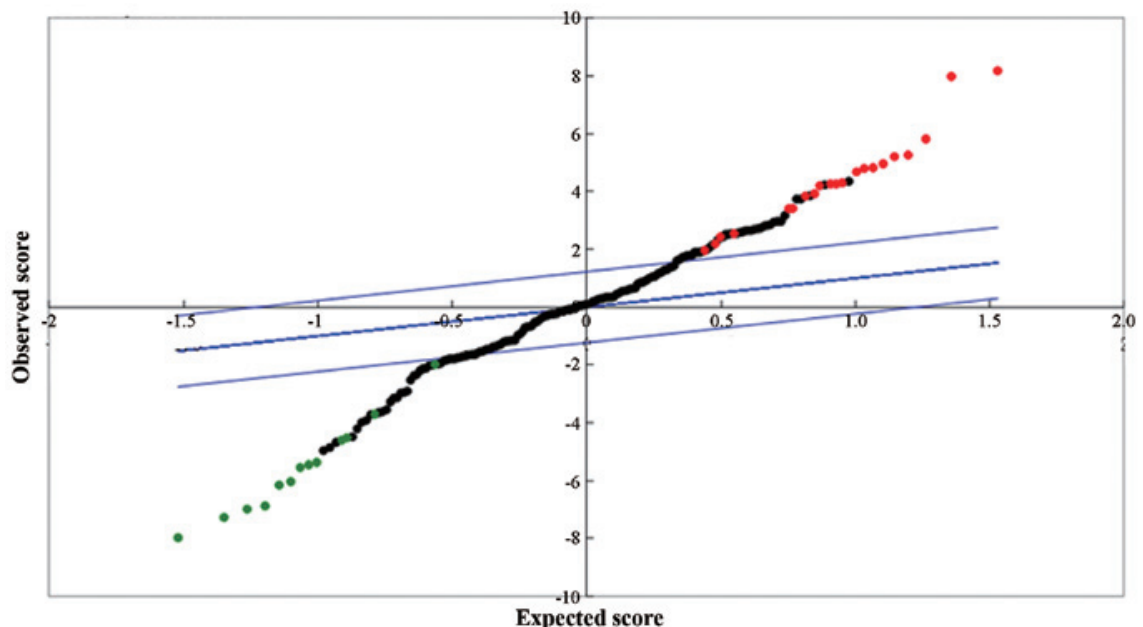

Figure 3. microRNA statistical scatter diagram results. The matrix was divided into 8 sub-arrays. Each array included 25 rows and 24 columns. The matrix spacing was $245 \mu \mathrm{m}$. The diameter of each dot was $\sim 150 \mathrm{iim}$. Each probe was repeated three times. Red color represented Cy5, while green color represented Cy3. If the Cy3 signal was stronger than the Cy5 signal, the dot was green, whereas if the Cy5 signal was stronger, the dot was red. If the intensity of the Cy3 and Cy5 signals was the same, the resulting dot was yellow. Cy, cyanine.

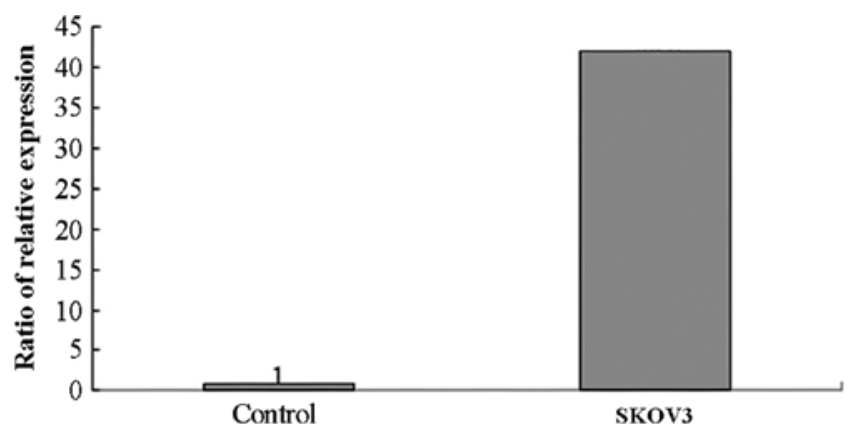

Figure 4. Reverse transcription-quantitative polymerase chain reaction revealed high expression of microRNA-30a-5p in SKOV3 cells following transfection for $24 \mathrm{~h}$.

anti-scramble miRNAs (Ambion; Thermo Fisher Scientific, Inc.) was $30 \mathrm{nmol} / \mathrm{l}$. The cellular total miRNAs were extracted after transfection for 24 and $48 \mathrm{~h}$.

3-(4,5-dimethylthiazol-2-yl)-2,5-diphenyltetrazolium bromide (MTT) assay. Cells were digested into a monoplast suspension with trypsin after transfection for $24 \mathrm{~h}$. The density was adjusted to $1 \times 10^{4}$ cells/ml and inoculated in a 96-well plate,

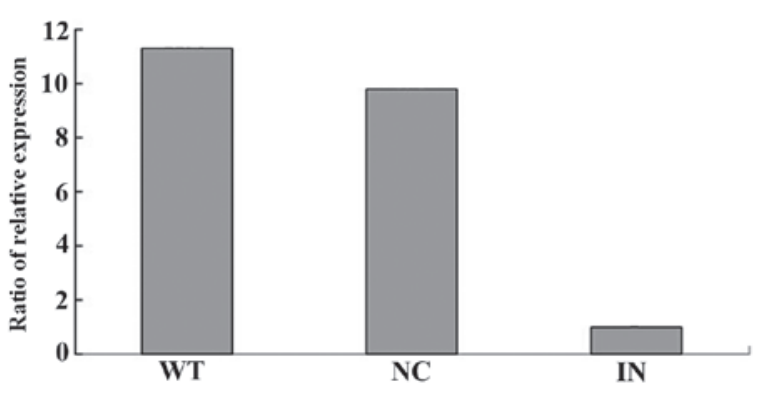

Figure 5. Reverse transcription-quantitative polymerase chain reaction was used to detect the expression of miR-30a-5p in SKOV3/DDP cells after transfection with a pre-miR inhibitor for $24 \mathrm{~h}$. miR,, microRNA; WT, wild-type; $\mathrm{NC}$, negative control; IN, inhibitor.

(100 $\mu 1 /$ well). Control groups were established prior to and following transfection. The cells in the 96-well plate were changed into the prepared concentration gradient of cisplatin in culture medium $24 \mathrm{~h}$ later. Five wells were set for each concentration, and the cells were cultured for additional $48 \mathrm{~h}$. Next, $10 \mu$ l MTT was added in each well, and the absorbance of each well was detected at $490 \mathrm{~nm}$ wavelength after being incubated for $4 \mathrm{~h}$ in the dark. The median lethal concentration 

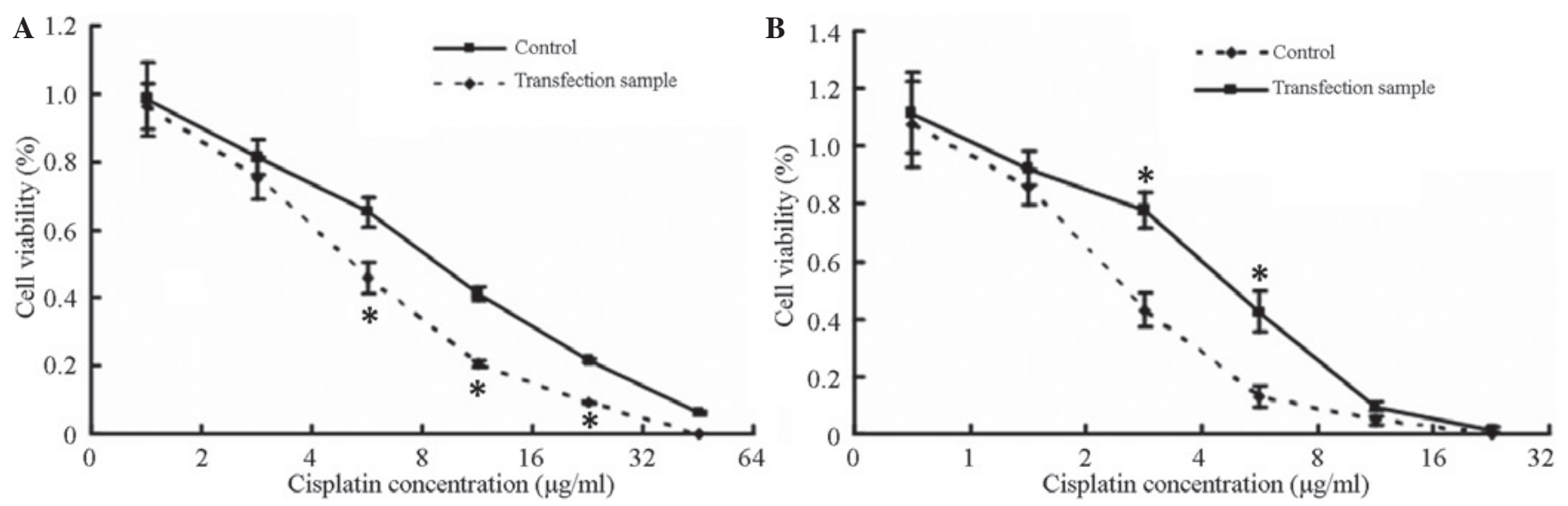

Figure 6. Effect of miR-30a-5p transfection on SKOV3/DDP and SKOV3 cells drug resistance. (A) SKOV3/DDP cells exhibited reduced resistance to cisplatin after transfection with inhibitor. (B) SKOV3 cells exhibited increased resistance to cisplatin after pre-miR transfection. " $\mathrm{P}<0.05$ vs. control cells. miR, microRNA.
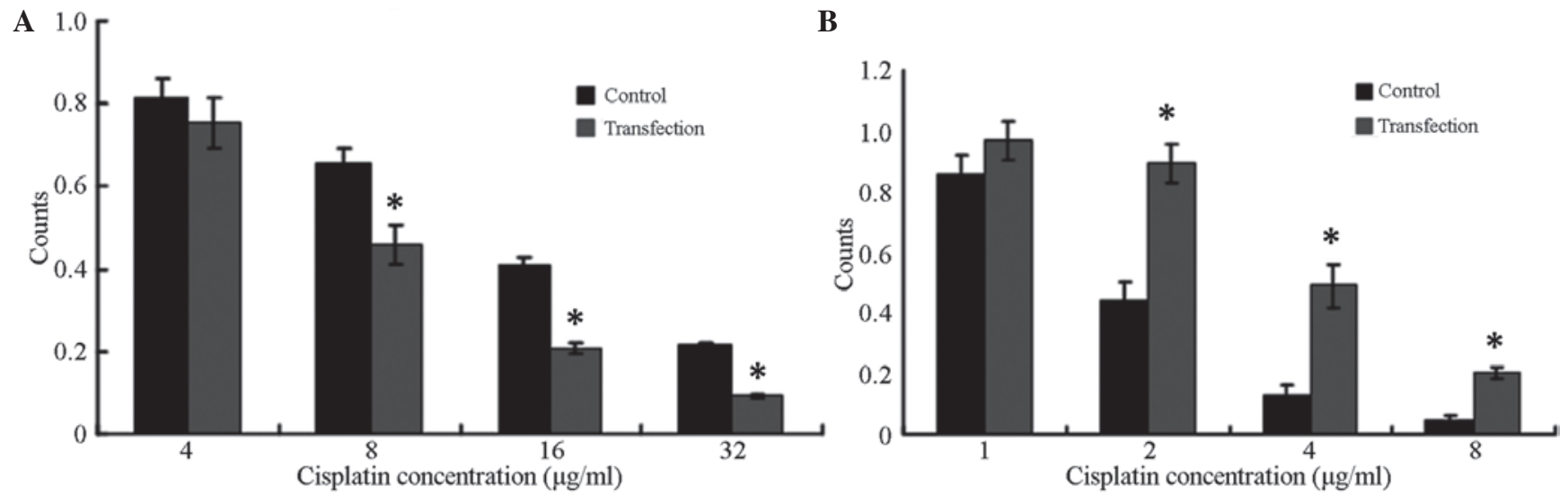

Figure 7. Effect of microRNA-30a-5p on SKOV3/DDP and SKOV3 cell activity. (A) Different concentrations of cisplatin were inoculated after transfection of SKOV3/DDP cells with inhibitor for $24 \mathrm{~h}$. Cell activities were reduced after SKOV3/DDP cells after inhibitor transfection. (B) Different concentrations of cisplatin were inoculated after transfection of SKOV3 cells with inhibitor for $24 \mathrm{~h}$. Cell activities were increased after SKOV3 cells after pre-miR transfection. ${ }^{*} \mathrm{P}<0.05$ vs. control cells.

$\left(\mathrm{IC}_{50}\right)$ of cisplatin was calculated. Each experiment was repeated three times, and five wells were set each time.

Statistical analysis. SPSS version 13.0 software (SPSS, Inc., Chicago, IL, USA) was used for statistical analysis. Data are presented as the mean \pm standard error of the mean. Student's t-test was used to compare the mean value between two groups. $\mathrm{P}<0.05$ was considered to indicate a statistically significant difference.

\section{Results}

Identification of mRNA extraction quality. RNA electrophoresis results are shown in Fig. 1. Bands corresponding to $5.8 \mathrm{~S}, 18 \mathrm{~S}$ and $28 \mathrm{~S}$ were clearly visible, indicating that non-biodegradable mRNA was obtained. The electrophoresis results revealed that the band brightness of RNA $28 \mathrm{~S}$ was more close to $1: 1$ than that of $18 \mathrm{~S}$ mRNA. Thus, the quality of the mRNA extracted met the experimental requirements, and the miRNA chip experiment could subsequently be performed.

Differences in expression profile analyzed by miRNA microarray chip. miRNA expression in drug-resistant and drug-sensitive ovarian cancer cells was detected by gene chip technology. Fig. 2 describes the miRNA chip hybridization results obtained, while Fig. 3 represents the results of miRNA statistical scatter diagram.

Compared with the drug-sensitive ovariancancer SKOV3 cell line, 19 miRNAs, including hsa-miR-99a-5p, hsa-miR-30a-5p, hsa-miR-34c-5p, hsa-miR-31-3p and hsa-miR-181d, were highly expressed in the drug-resistant SKOV3/DPP cell line, while 20 miRNAs, including hsa-miR-96-5p, hsa-miR-193b-3p and hsa-miR-200c-3p, were lowly expressed. In addition, 22 miRNAs, including hsa-miR-34a-5p, hsa-miR-30a-5p and hsa-miR-181c-3p, were highly expressed in the COC1/DPP cell line compared with the $\mathrm{COC} 1$ cell line, while 22 miRNAs, including hsa-miR-892b and hsa-miR-505-5p, were lowly expressed. Among them, both hsa-miR-30a-5p and hsa-miR-181 were highly expressed in the two cell lines.

Detection of miRNA expression by RT-qPCR. RT-qPCR was used to analyze the expression of miRNA-30a-5p in SKOV3/DDP and SKOV3 cells after transfection for $24 \mathrm{~h}$. The results revealed that the $\Delta \mathrm{Cq}$ of miRNA-30a- $5 \mathrm{p}$ was 2.3 after SKOV3 cell transfection with pre-miR for $24 \mathrm{~h}$, while the $\Delta \mathrm{Cq}$ was 7.7 in the negative control group. The relative 
expression level of miRNA-30a-5p was 42.2 times that of the negative control group after cell transfection with pre-miR for $24 \mathrm{~h}$. The RT-qPCR results also revealed that the $\Delta \mathrm{Cq}$ of miRNA-30a-5p was 8.9 after SKOV3/DDP cell transfection with pre-miR inhibitor for $24 \mathrm{~h}$, while the $\Delta \mathrm{Cq}$ of the negative control group was 5.6. The relative expression of miRNA-30a-5p was 9.8 times that of the negative control group after cell transfection with pre-miR inhibitor for $24 \mathrm{~h}$ (Figs. 4 and 5).

Effect of miRNA-30a-5p transfection on SKOV3/DDP and SKOV3 cells drug resistance. SKOV3/DDP cells exhibited reduced resistance to cisplatin after transfection with inhibitor. The $\mathrm{IC}_{50}$ value was $7.6 \pm 0.38 \mu \mathrm{g} / \mathrm{ml}$ in these cells compared with $13 \pm 0.47 \mu \mathrm{g} / \mathrm{ml}$ in the negative control group. The difference between the two groups was significant $(\mathrm{P}<0.05)$ (Fig. 6A).

SKOV3 cells exhibited increased resistance to cisplatin after pre-miR transfection $\left(\mathrm{IC}_{50}=3.5 \pm 0.26 \mu \mathrm{g} / \mathrm{ml}\right.$ vs $1.8 \pm 0.12 \mu \mathrm{g} / \mathrm{ml}$ for the negative control group). The difference between the two groups was significant $(\mathrm{P}<0.05)$ (Fig. 6B).

Effect of miRNA-30a-5p on SKOV3/DDP and SKOV3 cell activities. The cell number was significantly lower than that of the negative control group after $48 \mathrm{~h}$ of incubation with different concentrations of cisplatin following the transfection of SKOV3/DDP cells with inhibitor for $24 \mathrm{~h}$ cells $(\mathrm{P}<0.05)$. The cell activity was decreased significantly (Fig. 7A).

The cell number was significantly higher than that of the negative control group after $48 \mathrm{~h}$ of incubation with different concentrations of cisplatin following the transfection of SKOV3 cells with inhibitor for $24 \mathrm{~h}$ cells $(\mathrm{P}<0.05)$. The cell activity was increased significantly (Fig. 7B).

\section{Discussion}

A number of studies have demonstrated that miRNAs are involved in the occurrence and development of ovarian cancer (10-12). Zhang et al (16) reported that miRNA was involved in the occurrence and development of ovarian cancer drug resistance by studying a stem cell-like epithelial ovarian cancer line. Li et al (17) demonstrated that miRNA-27a was associated with ovarian cancer drug resistance, and the mechanism was that MDR1/P-GP gene regulated the HIPK2 gene.

Sorrentino et al (8) detected the expression of let-7e, miR-30C, miR-125b, miR-30a and miR-335 in the wild-type ovarian cancer cell line A2780, paclitaxel-resistant cell line A2780TAX and cisplatin-resistant cell line A2780CIS by chip technology. The authors observed that let-7e was overexpressed in A2780TAX cells, while miR-125b was lowly expressed in this cell line. Two miRNAs were inversely expressed in the other cell lines, while miR-30c, miR-130a and miR-335 were all lowly expressed in the above three cell lines (8). Therefore, it was inferred that let-7e had an inverse correlation with miR-125b, and that miRNA expression in ovarian cancer was associated with drug resistance of ovarian cancer, which was consistent with other studies (18-20).

In the present study, a miRNA microarray chip platform containing 924 probes was used for hybridization. The differentially expressed miRNAs between chemotherapy resistance and chemotherapy sensitivity in the ovarian cancer cell lines
SKOV3/DDP and SKOV3 were screened. Among them, 19 miRNAs, including hsa-miR-99a-5p, hsa-miR-30a-5p, hsa-miR-34c-5p, hsa-miR-31-3p and hsa-miR-181d, were observed to be highly expressed in SKOV3/DPP vs. SKOV3 cell lines, while 20 miRNAs, including hsa-miR-96-5p, hsa-miR-200c-3p and hsa-miR-193b-3p, were lowly expressed. In addition, 22 miRNAs, including hsa-miR-34a-5p, hsa-miR-30a-5p and hsa-miR-181c-3p, were observed to be highly expressed in COC1/DPP vs. COC1 cell lines, while 24 miRNAs, including hsa-miR-892b and hsa-miR-505-5p, were lowly expressed. Among them, both hsa-miR-30a-5p and hsa-miR-181 were highly expressed in the two cell lines. These findings may provide a theoretical basis and a novel approach to further study the association between miRNA and cisplatin resistance in ovarian cancer cells, as well as its possible mechanism.

miRNAs are likely to be important in the occurrence, development, diagnosis and treatment of ovarian cancer; thus, the number of studies on abnormal expression profiles of miRNA in serum and tissue of ovarian cancer patients has increased gradually $(3,21)$. The future goal should focus on accurately predicting ovarian cancer-associated miRNA target genes, studying the specific mechanism, and solving clinical diagnosis, treatment and prognosis evaluation-associated problems. The treatment perspective of cancer chemotherapy resistance with miRNA as a target is very wide. The studies on miRNA as a clinically therapeutic target are still in their initial stages. Since 1998, increasing attention has been paid to miRNA and the potential therapeutic effect of the corresponding exogenous small interfering RNA (22). miRNA treatment includes miRNA that substitutes low expression and miRNA that inhibits overexpression. Previous studies have used agomirs and antagomirs as analogues and inhibitors, respectively, of miRNAs for the treatment of cancer, and a large number of experiments in vivo have been conducted (23-27).

In conclusion, the present study confirmed that miRNA-30a-5p played a role in the drug-resistant mechanism of ovarian cancer. Future miRNA-30a-5p expression analysis may become an effective tool for predicting the effect of ovarian cancer chemotherapy. The mechanism of miRNA-30a-5p-mediated regulation of ovarian cancer drug resistance should be further investigated. Further studies will greatly promote the practical applications of miRNA in the prevention, diagnosis and treatment of ovarian cancer, which may provide a more broad application prospect in ovarian cancer-associated miRNA clinical studies.

\section{References}

1. Greenlee RT, Hill-Harmon MB, Murray T and Thun M: Cancer statistics, 2001. CA Cancer J Clin 51: 15-36, 2001.

2. Agarwal R and Kaye SB: Ovarian cancer: Strategies for overcoming resistance to chemotherapy. Nat Rev Cancer 3: 502-516, 2003.

3. Iorio MV, Visone R, Di Leva G, Donati V, Petrocca F, Casalini P, Taccioli C, Volinia S, Liu CG, Alder H, et al: MicroRNA signatures in human ovarian cancer. Cancer Res 67: 8699-8707, 2007.

4. Li L, Sun X, Wang $X$ and Ding C: WITHDRAWN: MicroRNA-200c and microRNA-141 as potential diagnostic and prognostic biomarkers for ovarian cancer. Biomed Pharmacother: pii: S0753-S3322; 00213-3, 2014. 
5. Banno K, Yanokura M, Iida M, Adachi M, Nakamura K, Nogami Y, Umene K, Masuda K, Kisu I, Nomura H, et al: Application of microRNA in diagnosis and treatment of ovarian cancer. Biomed Res Int 2014: 232817, 2014.

6. Liu L, Zou J, Wang Q, Yin FQ, Zhang W and Li L: Novel microRNAs expression of patients with chemotherapy drug-resistant and chemotherapy-sensitive epithelial ovarian cancer. Tumour Biol 35: 7713-7717, 2014.

7. Clop A, Marcq F, Takeda H, Pirottin D, Tordoir X, Bibé B, Bouix J, Caiment F, Elsen JM, Eychenne F, et al: A mutation creating a potential illegitimate microRNA target site in the myostatin gene affects muscularity in sheep. Nat Genet 38: 813-818, 2006.

8. Sorrentino A, Liu CG, Addario A, Peschle C, Scambia G and Ferlini C: Role of microRNAs in drug-resistant ovarian cancer cells. Gynecol Oncol 111: 478-486, 2008.

9. Singh SR and Rameshwar P (eds): MicroRNA in Development and in the Progression of Cancer. 1st edition. Springer, New York City, NY, 2014

10. Kinose Y, Sawada K, Nakamura K and Kimura T: The role of microRNAs in ovarian cancer. Biomed Res Int 2014: 249393 2014.

11. Kan CW, Howell VM, Hahn MA and Marsh DJ: Genomic alterations as mediators of miRNA dysregulation in ovarian cancer. Genes Chromosomes Cancer 54: 1-19, 2015.

12. Li Y, Yao L, Liu F, Hong J, Chen L, Zhang B and Zhang W: Characterization of microRNA expression in serous ovarian carcinoma. Int J Mol Med 34: 491-498, 2014.

13. Liu N, Zhou C, Zhao J and Chen Y: Reversal of paclitaxel resistance in epithelial ovarian carcinoma cells by a MUC1 aptamer-let-7i chimera. Cancer Invest 30: 577-582, 2012.

14. Lee IH, Park JB, Cheong M, Choi YS, Park D and Sin JI: Antitumor therapeutic and antimetastatic activity of electroporation-delivered human papillomavirus 16 E7 DNA vaccines: A possible mechanism for enhanced tumor control. DNA Cell Biol 30: 975-985, 2011

15. Nurkkala M, Wassén L, Nordström I, Gustavsson I, Slavica L, Josefsson A and Eriksson K: Conjugation of HPV16 E7 to cholera toxin enhances the HPV-specific T-cell recall responses to pulsed dendritic cells in vitro in women with cervical dysplasia. Vaccine 28: 5828-5836, 2010.
16. Zhang S, Lu Z, Unruh AK, Ivan C, Baggerly KA, Calin GA, Li Z, Bast RC Jr and Le XF: Clinically relevant microRNAs in ovarian cancer. Mol Cancer Res 13: 393-401, 2015.

17. Li Z, Hu S, Wang J, Cai J, Xiao L, Yu L and Wang Z: MiR-27a modulates MDR1/P-glycoprotein expression by targeting HIPK2 in human ovarian cancer cells. Gynecol Oncol 119: 125-130, 2010.

18. Luo J, Zhou J, Cheng Q, Zhou C and Ding Z: Role of microRNA-133a in epithelial ovarian cancer pathogenesis and progression. Oncol Lett 7: 1043-1048, 2014.

19. Zong C, Wang J and Shi TM: MicroRNA 130b enhances drug resistance in human ovarian cancer cells. Tumour Biol 35: 12151-12156, 2014.

20. Li B, Chen H, Wu N, Zhang WJ and Shang LX: Deregulation of miR-128 in ovarian cancer promotes cisplatin resistance. Int J Gynecol Cancer 24: 1381-1388, 2014.

21. Nam EJ, Yoon H, Kim SW, Kim H, Kim YT, Kim JH, Kim JW and Kim S: MicroRNA expression profiles in serous ovarian carcinoma. Clin Cancer Res 14: 2690-2695, 2008.

22. Zhang XY, Ding JX, Tao X and Hua KQ: FSH stimulates expression of the embryonic gene HMGA2 by downregulating let-7 in normal fimbrial epithelial cells of ovarian high-grade serous carcinomas. Exp Ther Med 5: 350-354, 2013.

23. Wan WN, Zhang YQ, Wang XM, Liu YJ, Zhang YX, Que YH, Zhao WJ and Li P: Down-regulated miR-22 as predictive biomarkers for prognosis of epithelial ovarian cancer. Diagn Pathol 9: 178, 2014

24. Chen S, Chen X, Xiu YL, Sun KX, Zong ZH and Zhao Y: MicroRNA 490-3P enhances the drug-resistance of human ovarian cancer cells. J Ovarian Res 7: 84, 2014

25. Zhu CL and Gao GS: miR-200a overexpression in advanced ovarian carcinomas as a prognostic indicator. Asian Pac J Cancer Prev 15: 8595-8601, 2014.

26. Zhao H, Liu S, Wang G, Wu X, Ding Y, Guo G, Jiang J and Cui S: Expression of miR-136 is associated with the primary cisplatin resistance of human epithelial ovarian cancer. Oncol Rep 33: 591-598, 2015.

27. Li L, He L, Zhao JL, Xiao J, Liu M, Li X and Tang H: miR-17-5p up-regulates YES1 to modulate the cell cycle progression and apoptosis in ovarian cancer cell lines. J Cell Biochem 116: 1050-1059, 2015 